\title{
Indicadores para evaluar el rendimiento de usuarios de oficina en clima templado cálido
}

\section{Indicators to evaluate the performance of office users in warm temperate climate}

$\underline{\text { Yesica Alamino Naranjo }}^{(*)}$, Ernesto Kuchen ${ }^{* *)}$

\section{RESUMEN}

El objetivo de este trabajo es encontrar rangos de rendimiento óptimo de usuarios de oficinas de tipología abierta-cerrada afectados por variables ambientales, y valorarlos a través de un indicador numérico. Para ello se lleva adelante un trabajo de campo en base a mediciones de variables ambientales como temperatura operativa, nivel de $\mathrm{CO} 2$, nivel de iluminación y nivel de ruido, y encuestas a usuarios durante tres periodos del año en 160 oficinas de tipo abierta-cerrada, en un edificio de referencia en clima templado cálido. Los resultados permiten conocer rangos de variabilidad preferidos y la valoración del rendimiento laboral por cada variable de estudio.

Palabras clave: Rendimiento laboral; oficina; calidad ambiental; confort.

\section{ABSTRACT}

The objective of this work is to find optimal performance ranges for users of open-closed typology offices affected by environmental variables, and assess them through a numerical indicator. For this, fieldwork is carried out in situ, based on measurements of environmental variables such as operative temperature, $\mathrm{CO} 2$ level, illumination level and noise level, and user surveys during three periods of the year, in 160 offices of type open-closed, in a reference building in a warm temperate climate. The results allow us to know the preferred ranges of variability and the assessment of work performance for each study variable.

Keywords: Work performance; office; indoor environmental; comfort.

(*) Dra. Arquitecta. Becaria Postdoctoral, Consejo Nacional de Investigaciones Científicas y Técnicas (CONICET), Buenos Aires (Argentina).

$\left.{ }^{* *}\right)$ Dr.-Ing. Arquitecto. Investigador, Consejo Nacional de Investigaciones Científicas y Técnicas (CONICET), Buenos Aires (Argentina).

Persona de contacto: y.alaminonaranjo@conicet.gov.ar (Y. Alamino Naranjo).

ORCID: https://orcid.org/oooo-0oo2-2325-2206 (Y. Alamino Naranjo); https://orcid.org/oooo-0oo1-6649-6932 (E. Kuchen).

Cómo citar este artículo/Citation: Yesica Alamino Naranjo, Ernesto Kuchen (2021). Indicadores para evaluar el rendimiento de usuarios de oficina en clima templado cálido. Informes de la Construcción, 73(564): e420. https://doi.org/10.3989/ic.83476

Copyright: (C) 2021 CSIC. Este es un artículo de acceso abierto distribuido bajo los términos de la licencia de uso y distribución Creative Commons Reconocimiento 4.0 Internacional (CC BY 4.0). 


\section{INTRODUCCIÓN}

Actualmente el diseño de espacios de oficina es pensado en busca de solucionar el problema del máximo aprovechamiento de superficies. Se pretende mejorar las condiciones interiores del edificio desde una mirada especulativa, bajo recortes de tipo económico, olvidando dar soluciones de calidad ambiental interior adecuadas para el Usuario Trabajador de oficina (UT), quien será el que lo habite durante la vida útil del edificio.

En términos laborales, la calidad de vida del UT toma relevancia, ya que una fuerza de trabajo confortable y saludable representa el componente vital de un negocio productivo y exitoso a largo plazo (1), (2). En este contexto, el costo del personal en salarios y beneficios es mayor al 90\% de los costos operativos de una empresa (3), con ello el rendimiento laboral (RL) toma una dimensión significativa en la situación financiera de empleadores.

En la sociedad actual el UT es demandado a ocupar más de un tercio del tiempo de una jornada laboral en espacios de oficina (4). Estos espacios se encuentran influenciados por factores ambientales que conforman la calidad ambiental interior (CAI). Desde el ámbito científico existen avances en la temática que permiten pensar sobre las variables ambientales interiores que ejercen mayor influencia sobre la salud y rendimiento en los espacios de oficina (5), entre ellos se destaca la temperatura (6), (7), (8); concentración de $\mathrm{CO}_{2}$ (9), (10); nivel lumínico (11), (12), (13) y nivel sonoro interior (14), (15).

Dichos avances muestran como la CAI puede influir en las capacidades cognitivas de las personas, su salud, actitudes y rendimiento, al mismo tiempo que permiten comprender problemas de diseño espacial en oficina. No obstante, se desconocen estudios donde se aborde el rendimiento laboral en oficina afectado por éstas cuatro variables de forma holística en clima templado cálido. De ello se desprende la importancia de profundizar el conocimiento en esta área considerada vacante.

El presente trabajo tiene como objetivo conocer la relación entre CAI en espacios de oficina y rendimiento laboral de los UT, mediante el estudio de cuatro variables ambientales interiores (temperatura operativa, nivel de iluminación, concentración de $\mathrm{CO} 2$ y nivel de ruido), con el objetivo de determinar rangos de rendimiento óptimo de usuarios de oficinas, que permitan la construcción de indicadores de rendimiento, a fin de su evaluación. Así mismo se reconocen dos tendencias tipológicas de oficina, abierta y cerrada, las cuales serán abordadas por separado en busca de encontrar posibles diferencias ambientales entre sí.

Para llevar adelante este trabajo se desarrolla un estudio de campo en el edificio "Centro Cívico" ubicado en la ciudad de San Juan, Argentina, durante tres periodos del año. La obtención de datos sobre el rendimiento de los UT se realiza a través de encuestas in situ. En paralelo se lleva a cabo la medición con sensores de temperatura $\left[{ }^{\circ} \mathrm{C}\right]$, concentración de $\mathrm{CO} 2$ [ppm $\left.\mathrm{CO} 2\right]$, nivel sonoro [dBA] y nivel lumínico [Lux].

\section{METODOLOGÍA}

Para llevar adelante la investigación se proponen una aproximación experimental mediante un trabajo de campo en oficinas ubicadas en la ciudad de San Juan, Argentina. Se llevan a cabo mediciones de tipo cuantitativas de variables ambientales de influencia térmica, calidad del aire, visual y acústica, y en forma simultánea, se indaga sobre la valoración del RL mediante preguntas de auto-reporte.

Este procedimiento se realiza durante una semana típica del periodo verano, transitorio e invierno en dos tipologías arquitectónicas de oficina clasificadas en abierta y cerrada.

De los resultados del presente trabajo, se construye indicadores de rendimiento laboral óptimo (IRLO) por tipología de oficina en cada periodo del año. El mismo se traduce a una escala cuantitativa y gráfica.

\subsection{Caracterización geográfica}

La ciudad de San Juan, Argentina se ubica a 630 metros sobre el nivel del mar, latitud $31,6^{\circ}$ Sur y longitud $68,5^{\circ}$ Oeste. El clima según normativa IRAM se corresponde a templado-cálido (16) con grandes variaciones de temperatura diarias y anuales, transparencia atmosférica y baja humedad. El régimen de lluvias es continental, con una frecuencia media baja. Según la clasificación de Köppen es de tipo BWK (clima árido templado y frío). Esta región posee una temperatura media anual de $17.2^{\circ} \mathrm{C}$ en el Valle de Tulum y superior a $18^{\circ} \mathrm{C}$ durante el mes más cálido Enero $>23^{\circ} \mathrm{C}(17)$. Presenta vientos frecuentes moderados del sector sud-este y viento "Zonda", seco-cálido calificado como "evento severo" del oeste, con ráfagas intensas (18). Este último es casi constante durante agosto y septiembre (19).

\subsection{Objeto de estudio}

Del conjunto de edificios emplazados en la Ciudad de San Juan, se procede a analizar aquellos que presentan características que los destaquen en relación al impacto ambiental que generan.

Se analizan los consumos energéticos de la población de edificios y su relación por metro cuadrado de superficie útil (climatizada), destinada a espacios de trabajo (oficinas), considerando aquellos que superan los 3 (tres) niveles. El edificio Centro Cívico (ver figura 1) es seleccionado por presentar el mayor consumo de energía eléctrica, con valores superiores a $\operatorname{los} 340 \mathrm{kWh} / \mathrm{m} 2$ año. El uso elevado de recursos energéticos en este edificio, permite mantener condiciones interiores, independientemente de la evolución del clima exterior y de las necesidades de los usuarios.

Entre los aspectos a destacar del edificio (ver tala 1), se reconoce en relación a las condiciones térmicas, la baja variabilidad de temperatura operativa independientemente de las estaciones del año. La figura 2 muestra la suma de frecuencia acumulada durante los tres periodos de estudio, pudiendo identificar una concentración significativa de mediciones entre $23 \mathrm{C}^{\mathrm{O}}$ a $26 \mathrm{C}^{\circ}$, valores definidos como rangos establecidos por la norma internacional ISO 7730 (20), para condiciones de confort térmico aceptable (Categoría $\mathrm{B}$ ) para período de verano (ver Tabla 2), y que se replican en los demás períodos. 


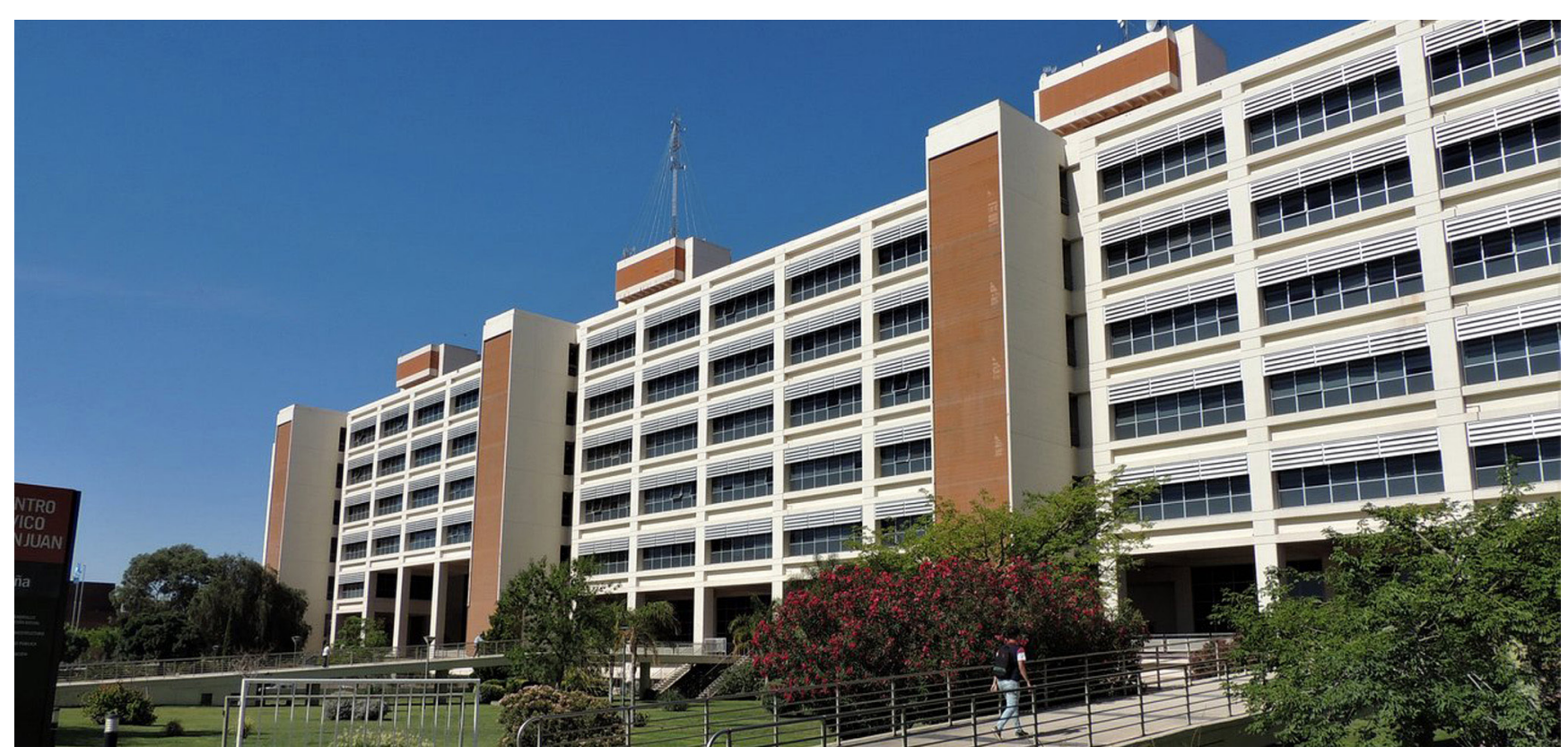

Figura 1. Fachada Este Edificio Centro Cívico. Fuente: Elaboración de los autores.

Tabla 1: Caracterización del edificio Centro Cívico Niveles. Fuente: Elaboración de los autores.

\begin{tabular}{|c|c|}
\hline Orientación & Este-Oeste \\
\hline Superficie total & $80.873 \mathrm{~m}^{2}$ \\
\hline Superficie de oficina [\%] & $59 \%$ \\
\hline Sistema de climatización frío & HVAC \\
\hline Sistema de climatización calor & HVAC \\
\hline Consumo [kWh/m ${ }^{2}$.año] & 342 \\
\hline Cantidad de UT & 4046 \\
\hline UT encuestados & 335 \\
\hline
\end{tabular}

La Categoría B representaría un PMV (Predicted Mean Vote) de $\pm 0,5$ en una escala que varía entre \pm 3 (mucho calor/ mucho frío) y un PPD (Predicted Percentage of Dissatisfied) de $10 \%$ o lo que es lo mismo, un 90\% de aceptación térmica, lo que implica un enorme empleo de recursos energéticos.

De considerar las exigencias de la norma (20) para invierno, más del 60\% de los valores de medición quedarían por encima del rango aceptable (ver Tabla 2), conduciendo un enorme consumo de recursos para contrarrestar los efectos ad- versos del clima de invierno. Esta observación indica que las condiciones térmicas interiores del edificio son controladas como si fuese una cámara climática, es decir, con condiciones interiores seteadas a valores relativamente constantes durante todo el año, sin importar la opinión, sensación o preferencia de los usuarios, ni sus niveles de rendimiento afectado por la variabilidad ambiental interior.

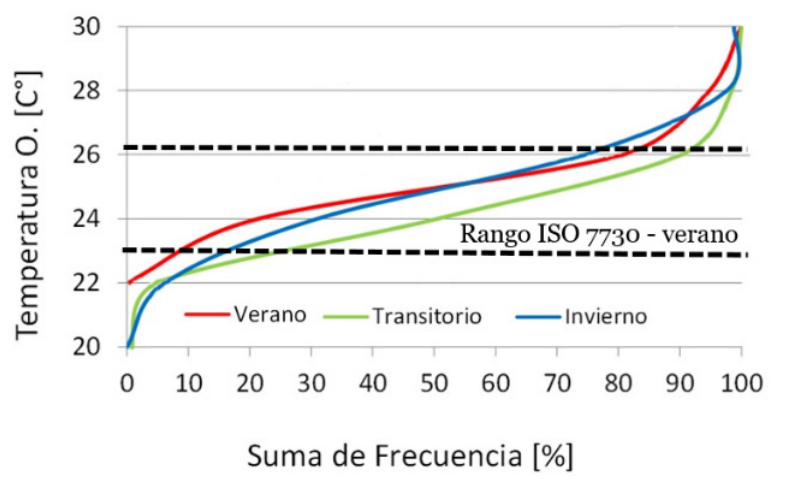

Figura 2. Suma de frecuencia de los datos medidos de temperatura operativa $\left(\mathrm{C}^{\circ}\right)$ según estaciones verano, transitorio, invierno. Fuente: Elaboración de los autores.

Tabla 2: Condiciones de confort térmico según Norma ISO 7730:2006, Categoría “B” (Confort aceptable).

\begin{tabular}{|c|c|c|}
\hline Condiciones & Invierno & Verano \\
\hline Temperatura Operativa & $20^{\circ}-24^{\mathrm{o}}-26^{\circ}$ \\
\hline Velocidad del Aire & $<0.16 \mathrm{~m} / \mathrm{s}$ & $<0.19 \mathrm{~m} / \mathrm{s}$ \\
\hline Humedad Relativa & $50 \%$ & $50^{\circ}$ \\
\hline Resistencia térmica de la vestimenta & 1 clo & 0.5 clo \\
\hline
\end{tabular}




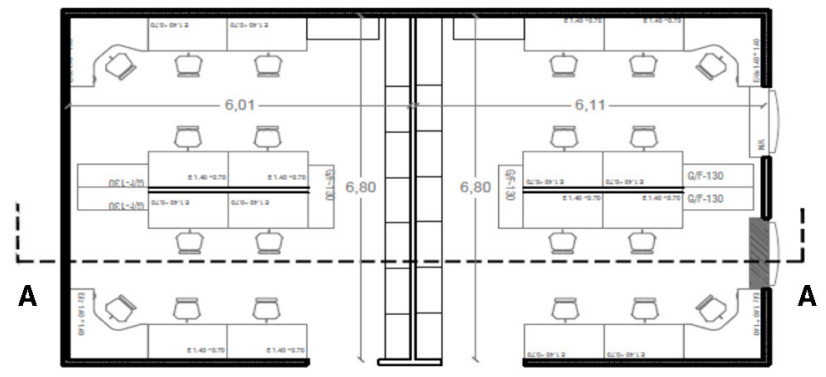

Figura 3. Planta tipo de dos oficinas de tipología arquitectónica abierta (OTAA). Fuente: Elaboración de los autores.

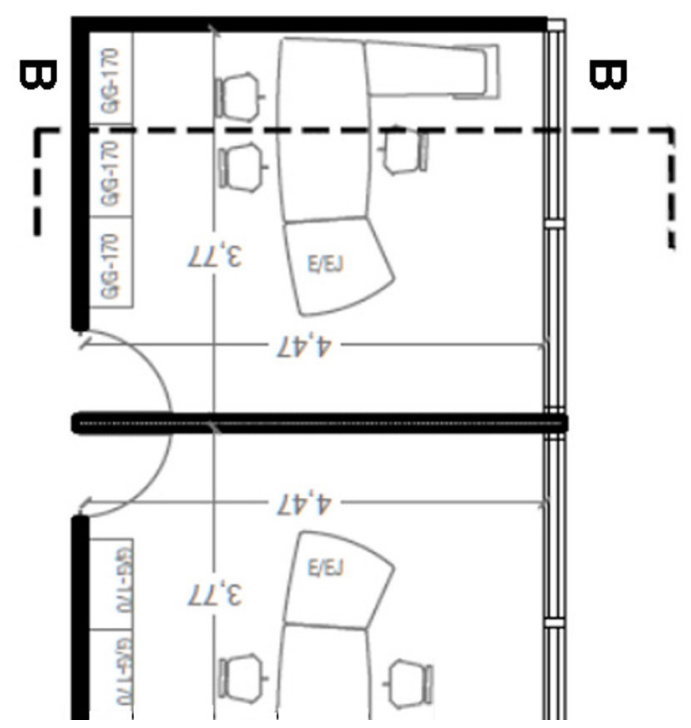

Figura 5. Planta tipo de dos oficinas de tipología arquitectónica cerrada (OTAC). Fuente: Elaboración de los autores.

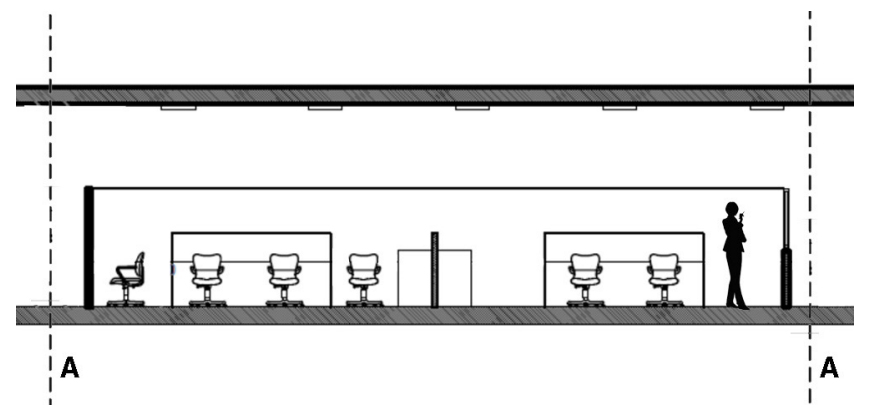

Figura 4. Corte tipo de dos oficinas de tipología arquitectónica abierta (OTAA). Fuente: Elaboración de los autores.

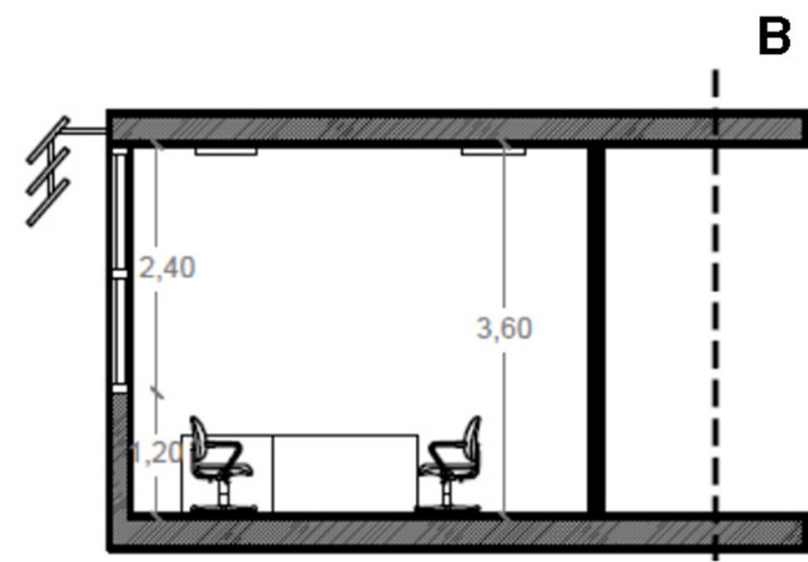

B

Figura 6. Corte tipo de oficina de tipología arquitectónica cerrada (OTAC). Fuente: Elaboración de los autores.

Tabla 3: Caracterización tipológica- arquitectónica de oficina. Fuente: Elaboración de los autores.

\begin{tabular}{|c|c|c|}
\hline Caracterización & OTAC & OTAA \\
\hline Presencia de Ventanas & $\mathrm{si}$ & no \\
\hline Control de Iluminación natural & $\mathrm{si}$ & no \\
\hline Altura de panel-cerramiento & $3,60 \mathrm{~m} .(100 \%)$ & 0.80 $\mathrm{m}-2.10 \mathrm{~m} .(25 \%)$ \\
\hline Factor de ocupación promedio & $5.10 \mathrm{~m} 2 /$ persona & 3 a 11 personas \\
\hline Capacidad de personas & 2 a 6 personas & Trabajo interno-Atención al público \\
\hline Actividad & Trabajo interno &
\end{tabular}

La variabilidad de la calidad ambiental interior exige distinguir elementos y agruparlos según sus características. Es por ello que en este trabajo los espacios de oficina son diferenciados en dos tipos: oficina de tipología arquitectónica abierta (OTAA) (ver figura 3 y figura 4) y oficina de tipología arquitectónica cerrada (OTAC) (ver figura 5 y figura 6). Ambas presentan diferencias destacables que llevan a establecer ventajas de OTAC sobre OTAA. La tabla 3 muestra las características que permiten establecer las principales diferencias consideradas en este trabajo.

\subsection{Procedimiento metodológico}

Se basa en un trabajo de campo a través de mediciones con sensores y encuestas a usuarios. La metodología de medición consiste en diseñar una forma sistemática de recolección de datos objetivos (sensores) y en simultáneo de datos subjetivos (encuestas) llevados a cabo durante una semana representativa en tres períodos del año: invierno (PIn), transitorio (PTr) y verano (PVe). Las 160 oficinas seleccionadas para el relevamiento se distribuyen aleatoriamente en cantidades representativas de OTAA y OTAC. Se realizan 335 encuestas a 
UT por periodo estacional, y en su conjunto, 1005 durante el año 2019. Para el levantamiento de datos se usa la sistemática del tipo "Spot" (focalizada), basada en las técnicas desarrolladas en otras latitudes (21), (22) y se adapta a la necesidad del relevamiento de los cuatro parámetros ambientales. Para ello se diseña una unidad móvil (UM) de obtención de datos que permite trasladar los 5 (cinco) instrumentos de medición dentro del edificio a fin de concretar el relevamiento de la mayor cantidad de espacios posibles en un tiempo acotado (ver figura 7).

La UM consiste en una caja desmontable, con dispositivos móviles, compartimento para el traslado del instrumental de medición y espacio para la instalación de baterías para el suministro de carga durante un relevamiento continuo y prolongado. Los sensores para el relevamiento de los parámetros: temperatura operativa, concentración de dióxido de carbono, nivel de ruido y nivel de iluminación, se muestran en figura 8. Cada sensor permite recolectar valores de las variables que afectan en diferentes grados la confortabilidad y el rendimiento de los usuarios trabajadores. Entre ellos se identifican:

1. Calidad del aire: sensor tipo TELAIRE modelo 7001. Permite medir niveles de $\mathrm{CO}_{2}(\mathrm{ppm})$ en un rango de o a 2500 ppm en tiempo real. Posee sensibilidad: \pm 1 ppm y precisión: \pm 50 ppm de la lectura.

2. Confort visual: sensor tipo Luxómetro modelo YK-2005LX. Permite medir Niveles de iluminación (lux) en plano de trabajo en un rango de 000/100, ooo Lux en tiempo real, sensibilidad espectral se corresponde con las exigencias de la curva CIE (International Commission on Illumination) y precisión de $\pm 4 \%+2$ dígitos)

3. Confort térmico: sensor tipo HOBO modelo U12-006. Permite medir temperatura $\left(\mathrm{C}^{\circ}\right)$ del aire en un rango de $-40 \mathrm{a}+100 \mathrm{C}^{\circ}$, con precisión de $\pm 0,5^{\circ} \mathrm{Ca} 20^{\circ} \mathrm{C}$, en condiciones de humedad de 5 a $95 \%$ H.r. sin condensar. Para la medición necesita un tiempo de estabilización entre 4 a 5 minutos (en aire estático)

4. Confort térmico: Pistola Infrarroja laser tipo Ajavision modelo $\mathrm{WH}_{3} 80$. Permite medir temperatura radiante media $\left(\mathrm{C}^{\circ}\right)$ en un rango de $-50^{\circ} \mathrm{C}+380^{\circ} \mathrm{C}$. Posee precisión de $\pm 3^{\circ} \mathrm{C}$.

5. Confort acústico: sensor tipo Decibelímetro modelo SL4023 SD. Permite medir niveles sonoros (dB) en rango automático: 30 a $130 \mathrm{~dB}$. y en rango manual ( 3 rangos): de 30 a $80 \mathrm{~dB}, 50$ a $100 \mathrm{~dB}, 80$ a $130 \mathrm{~dB}$. Ponderación de tiempo: rápido/lento. Ponderación de frecuencia de A (dBA)/ C (dBC). La medición llevada a cabo en el presente trabajo se realizó en un rango de 50 a $100 \mathrm{~dB}$, con ponderación de tiempo lento y ponderación de frecuencia $\mathrm{A}$.

La variabilidad del clima interior en relación a la influencia del clima exterior en tanto aspectos térmicos, lumínicos (presencia de la luz solar), acústicos y de calidad de aire por la posibilidad de ventilar mediante apertura de ventanas, exige tomar mediciones en períodos estacionales diferentes. La medición comienza al posicionar la UM dentro de una oficina y junto a un espacio de trabajo (escritorio), que se encuentra ocupado por un UT (ver figura 7). Los sensores se encuentran midiendo constantemente, posicionados fijos en la UM a una misma altura de aproximadamente 1,10 metro del nivel de piso interior, a una distancia máxima de $1 \mathrm{~m}$ y mínima de o,5m del UT del espacio de trabajo evaluado, posibilitando captar las condiciones ambientales percibidas por el sujeto.

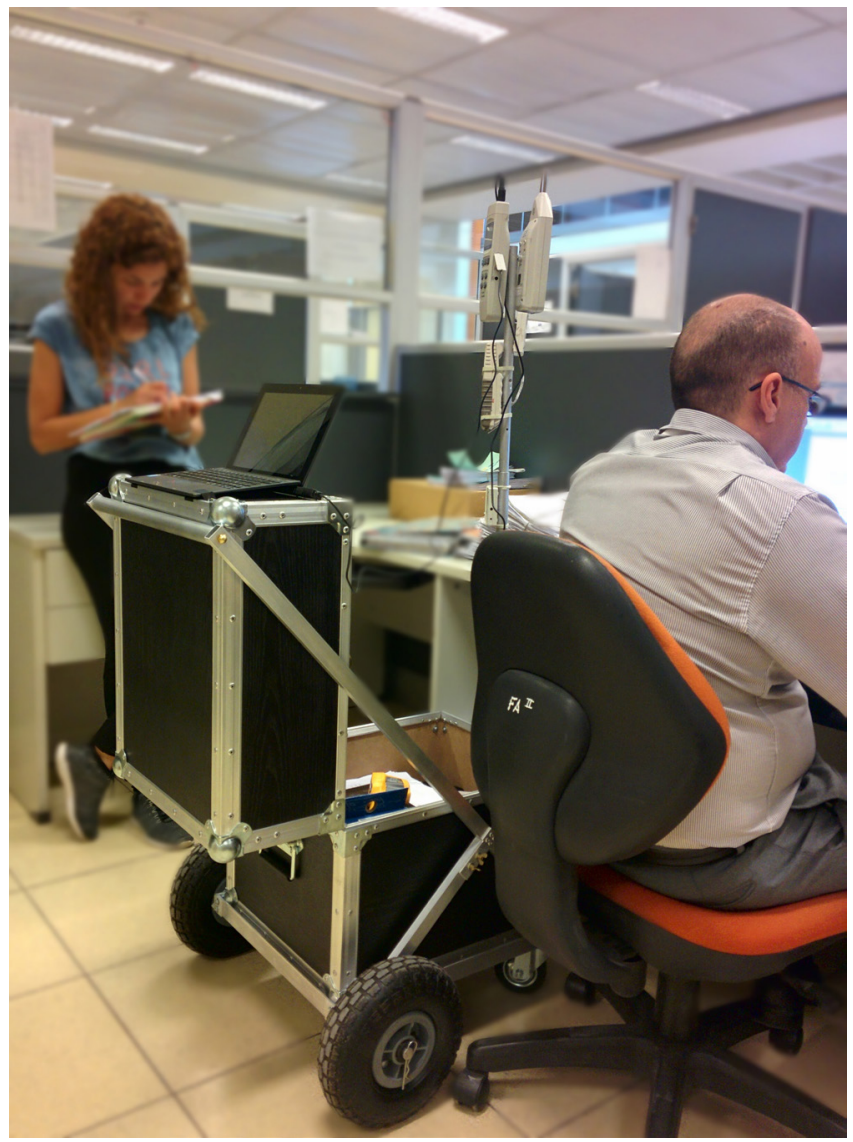

Figura 7. Unidad móvil (desplegada y funcionando). Fuente: Elaboración de los autores.

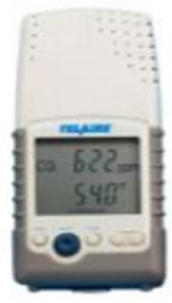

[1]
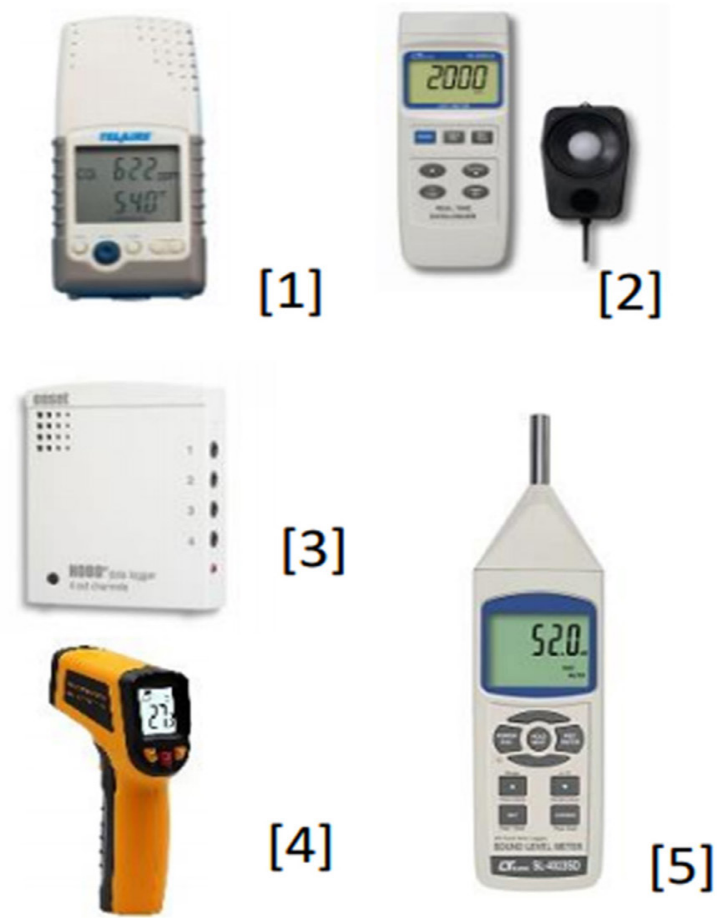

Figura 8. Sensores confort/rendimiento. Fuente: Elaboración de los autores. 
Los primeros cinco minutos se corresponden con la etapa de "aclimatación" de los sensores. Durante los cinco minutos siguientes se realiza el registro de las condiciones ambientales de cada oficina mediante una planilla donde el asistente de medición releva entre otros: apertura de ventanas, existencia de corrientes de aire, uso de parasoles.

Durante este periodo el usuario responde la encuesta test (2 páginas). Dicha encuesta permite obtener información subjetiva proveniente del UT. La misma es de tipo exploratoria ya que permite adquirir información para la estructuración de muestras y cálculos estadísticos, y descriptivas ya que logra caracterizar una parte de la población.

De la información lograda a partir del uso del cuestionario, se destaca la obtención de un diagnóstico del UT respecto a los parámetros de influencia.

Entre las preguntas realizadas toma mayor relevancia aquellas que indagan sobre el Voto de Rendimiento (VR) del UT. Las mismas se basan en la pregunta de auto-reporte desarrollada por los autores Humphreys y Nicol (23), donde se consulta de forma explícita "¿En qué medida (o\% - 100\%) siente que la calidad ambiental afecta negativamente a su rendimiento?”. En base a esto, se diferencian aquellas secciones que se refieren al confort térmico, calidad del aire, confort lumínico y confort sonoro. Cada una de estas secciones de la encuesta presenta un vínculo con cada sensor de medición ya que en la etapa de análisis ambos datos, subjetivos (de la encuesta) y objetivos (del sensor) se estudian correlacionados.

El tiempo necesario para responder el cuestionario por el UT, maniobrar la unidad móvil dentro de una oficina y examinar el espacio de estudio, dura no más de diez minutos. Cinco minutos adicionales se suponen para desplazar la UM dentro del edificio hasta otra oficina. Así, es posible examinar 4 (cuatro) espacios de oficina por hora.

\subsection{Método de análisis}

Los rangos de rendimiento se construyen como medio para obtener un indicador IRLO del UT en sus respectivos espacios de trabajo (TOAA-TOAC) y periodos del año (PVe, PTr, PIn).

Para lograrlo se llevan a cabo los siguientes pasos:

1. Se contabilizan los valores de cada variable ambiental en que el UT auto-reporta influencia nula (o\%) sobre su rendimiento.

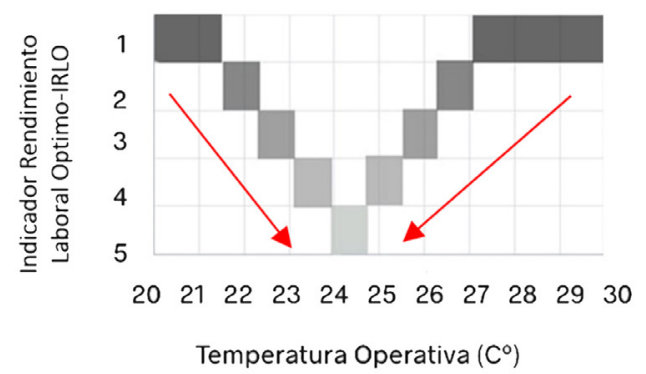

Figura 9. Gráfico de valoración de rangos de rendimiento según equivalente numérico de 1 (malo) a 5 (excelente), para variable ambiental térmica de dos polos de disconformidad (ejemplo). Fuente: Elaboración de los autores.
2. Se definen valores máximos y mínimos del Voto de Rendimiento/Térmico (VRt), del Voto de Rendimiento/Calidad de Aire (VRa), Voto de Rendimiento/ Nivel Iluminación (VRi) y Voto de Rendimiento/Ruido (VRr) que determinan la máxima variabilidad posible de cada parámetro ambiental de influencia.

3. Se definen rangos intermedios en torno a la división entre valor óptimo ( $\mathrm{VR}=0 \%$ ) y valor máximo, y división entre valor óptimo $(\mathrm{VR}=\mathrm{O} \%)$ y valor mínimo.

4. Para la obtención de IRLO se definen equivalentes numéricos e intervalos de puntuación a fin de establecer la valoración cualitativa de cada rango, desde "excelente" con un IRLO igual a 5, hasta "malo" con un IRLO igual a 1, para VRt, VRa, VRi y VRr, según tabla 4.

Tabla 4: Equivalentes numérico de rangos de rendimiento-IRLO. Fuente: Elaboración de los autores.

\begin{tabular}{|c|c|}
\hline $\begin{array}{c}\text { Valoración } \\
\text { cualitativa }\end{array}$ & $\begin{array}{c}\text { Indicador de Rendimiento Laboral } \\
\text { Optimo (IRLO) }\end{array}$ \\
\hline Excelente & 5 \\
\hline Muy Bueno & 4 \\
\hline Bueno & 3 \\
\hline Regular & $\mathbf{2}$ \\
\hline Malo & $\mathbf{1}$ \\
\hline
\end{tabular}

\section{RESULTADOS Y DISCUSIÓN}

Los rangos construidos para VRt, VRa, VRi y VRr, por cada variable de estudio se sintetizan en gráficos de ejes cartesianos a fin de lograr una fácil lectura en la evaluación del RL. El gráfico propuesto en la figura 9 y figura 10, se compone de un par de ejes cartesianos, donde en el eje de abscisas se ubican valores de medición de cada variable ambiental, entre ellos, temperatura $\left[\mathrm{C}^{\circ}\right]$, concentración de $\mathrm{CO} 2$ [ppm-CO2], niveles de iluminación [Lux] y niveles sonoros [dBA]; mientras que, en el eje de ordenada, se disponen los IRLO de la variable de análisis a través del equivalente numérico según denominación en tabla 4.

La mayor o menor amplitud de los rangos se asocia a la capacidad de adaptación del UT respecto a la variable correspondiente. En los casos ejemplos citados en este apartado, la variable ambiental podrá tener 2 (dos) polos de disconformidad (ver figura 9) o 1 (un) polo de disconformidad (ver figura 10). Las flechas sobre los casos ejemplo muestran la tendencia hacia una mejora en los IRLO.

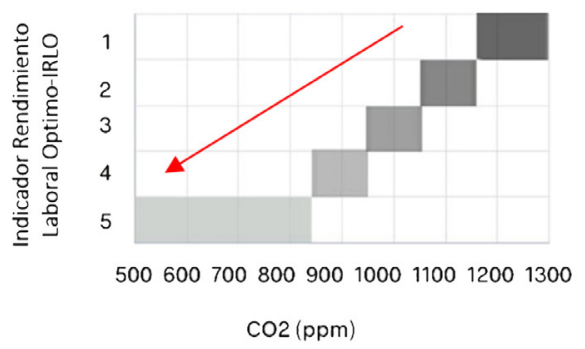

Figura 10. Gráfico de valoración de rangos de rendimiento según equivalente numérico de 1 (malo) a 5 (excelente), para variable ambiental calidad de aire de un polo de disconformidad (ejemplo). Fuente: Elaboración de los autores. 


\subsection{Variable temperatura}

La temperatura operativa (Top) afecta con su variabilidad al confort térmico en espacios interiores. La misma es medida a partir de combinar la temperatura del aire y la radiante media obteniendo un promedio. Dichos valores son medidos en grados Celsius $\left(\mathrm{C}^{\circ}\right)$, y su variabilidad no depende de la apertura de ventanas, ya que no está permitida su manipulación durante la jornada de trabajo por normativas internas del edificio, no obstante, se encontraron casos de estudio, donde los UT operaron las mismas en virtud de mejoras ambientales internas.

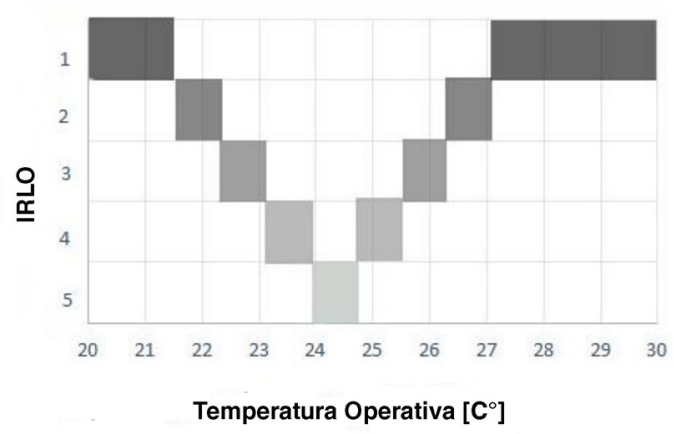

Figura 11. Rangos de variabilidad del RL influenciado por la temperatura durante PVe en OTAA.

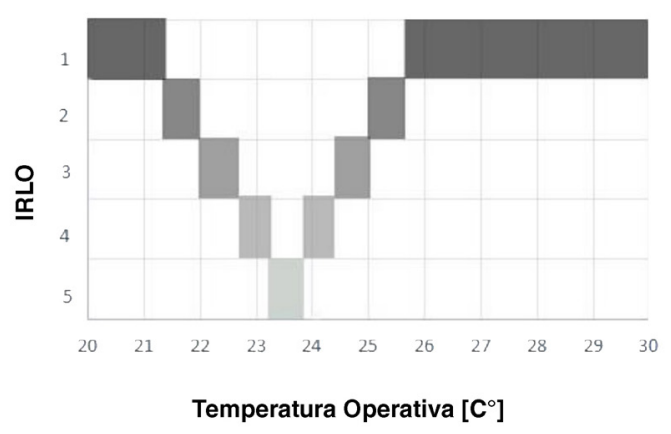

Figura 13. Rangos de variabilidad del RL influenciado por la temperatura durante PTr en OTAA.

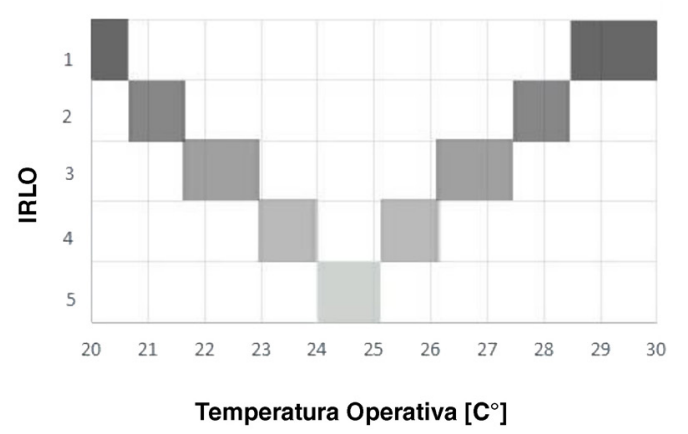

Figura 15. Rangos de variabilidad del RL influenciado por la temperatura durante PIn en OTAA.
Las siguientes figuras muestra la clasificación de rangos de rendimiento obtenidos por periodo de estudio.

Durante los periodos extremos (PVe y PIn) los rangos de RL encontrados en tipología OTAA, poseen mayor amplitud respecto a los rangos en OTAC (ver figura 11, 12, 15, 16). Estos valores revelan la mayor capacidad de adaptación térmica que posee el UT de OTAA respecto al UT de OTAC.

Seguidamente se observa en PTr (ver figuras 13 y figura 14) una mayor preferencia a trabajar con temperaturas más elevadas en UT de OTAC, existiendo una diferencia promedio de $1 \mathrm{C}^{\mathrm{o}}$ para todos los niveles de IRLO.

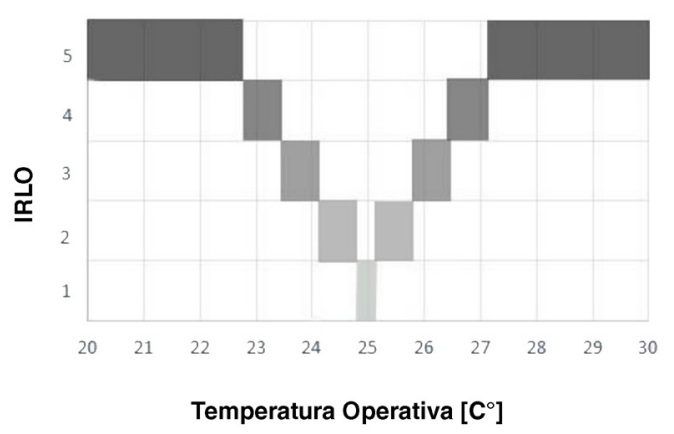

Figura 12. Rangos de variabilidad del RL influenciado por la temperatura durante PVe en OTAC.

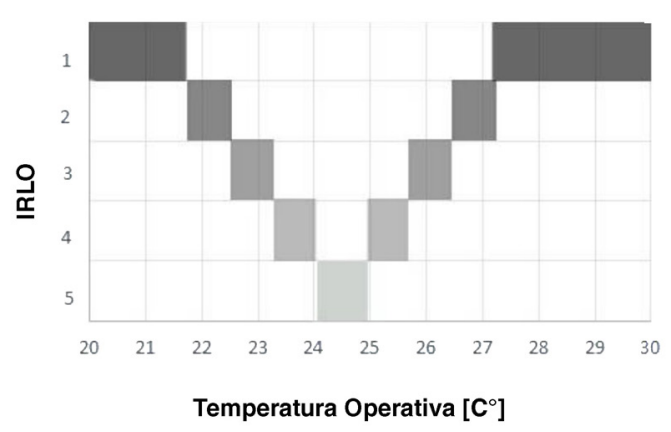

Figura 14. Rangos de variabilidad del RL influenciado por la temperatura durante PTr en OTAC

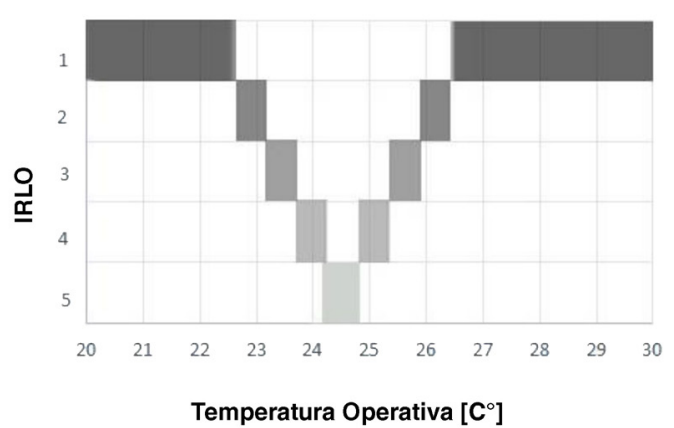

Figura 16. Rangos de variabilidad del RL influenciado por la temperatura durante PIn en OTAC. 


\subsection{Variable calidad de aire}

La calidad del aire es medida según los niveles de concentración de dióxido de carbono $\left(\mathrm{CO}_{2}\right)$ presentes en aire. Dichos niveles dependientes de la presencia de personas y del porcentaje de aire renovado podrían afectar la confortabilidad del UT y con ello su rendimiento en las tareas de oficina. Los niveles de $\mathrm{CO}_{2}$ se miden en ppm (partes por millón) en cada espacio analizado, y su variabilidad no depende de la apertura de ventanas, ya que no está permitida su manipulación durante la jornada de trabajo por normativas internas del edificio, no obstante, se encontraron casos donde los UT operaron las misma en busca de mejoras ambientales internas.

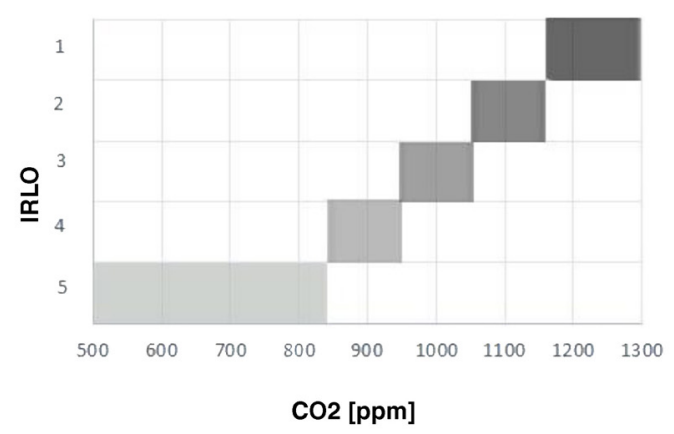

Figura 17. Rangos de variabilidad del RL influenciado por niveles de $\mathrm{CO} 2$ durante PVe en OTAA.

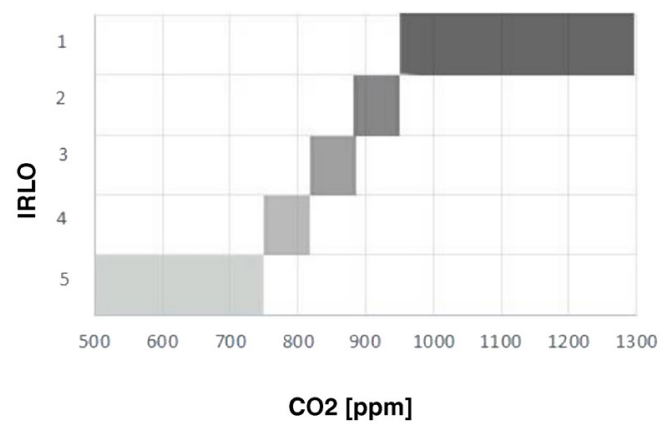

Figura 19. Rangos de variabilidad del RL influenciado por niveles de $\mathrm{CO} 2$ durante PTr en OTAA.

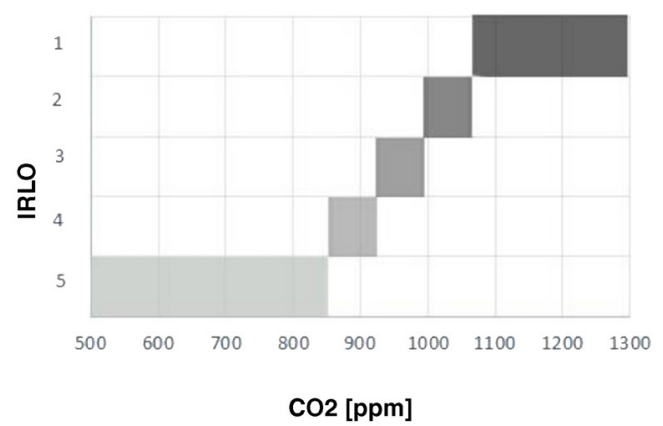

Figura 21. Rangos de variabilidad del RL influenciado por niveles de $\mathrm{CO} 2$ durante PIn en OTAA.
Las siguientes figuras muestran la clasificación de rangos de rendimiento obtenidos por periodo de estudio.

En el análisis comparativo entre tipologías de oficina (ver figura 17 a 22), se observa en IRLO=5 mayor amplitud de los rangos de RL en OTAA respecto a OTAC. Esta situación se mantiene durante los tres periodos de estudio.

La amplitud de dichos rangos permite identificar a un UT de OTAA con mayor capacidad de adaptación a mayores valores de concentración de $\mathrm{CO}_{2}$ es decir, valores entre 750 a 840 ppm (ver figura 17, 19 y 21) según periodo, sin ver afectado su rendimiento. Este rango es menor para OTAC, admitiendo niveles de $\mathrm{CO}_{2}$ entre 627 a 687 ppm.

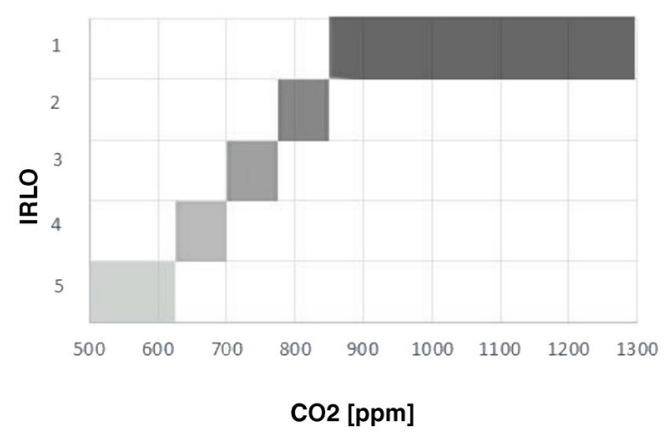

Figura 18. Rangos de variabilidad del RL influenciado por niveles de $\mathrm{CO} 2$ durante PVe en OTAC.

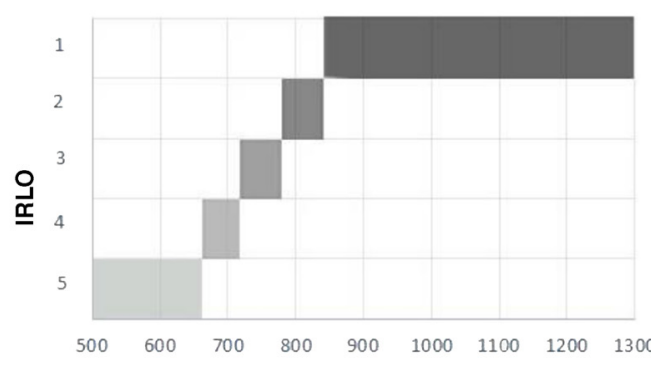

CO2 [ppm]

Figura 20. Rangos de variabilidad del RL influenciado por niveles de CO2 durante PTr en OTAC.

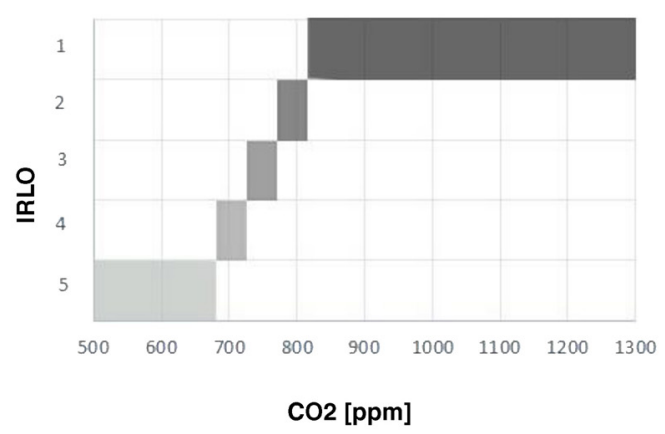

Figura 22. Rangos de variabilidad del RL influenciado por niveles de $\mathrm{CO} 2$ durante PIn en OTAC. 


\subsection{Variable nivel de iluminación}

El confort lumínico es medido en términos de niveles de iluminancia sobre el plano de trabajo sin considerar la fuente de iluminación (natural o artificial). Los mismos son medidos en Lux.

Las siguientes figuras muestran la clasificación de rangos de rendimiento obtenidos por periodo de estudio.

De la observación de rangos de rendimiento afectado por los niveles de iluminación interior entre tipos de oficina, se des- taca para el IRLO $=5$ (excelente) una diferencia lumínica con valores superiores en OTAC respecto a OTAA. Esta característica se da en PVe, con una diferencia promedio de 100 lux (figura 23 y 24). En PTr estos valores aumentan, existiendo una diferencia promedio de 175 lux (figura 25 y 26), mientras que en PIn disminuye a 48 lux (figura 27 y 28).

El comportamiento de los datos permite determinar que los UT de OTAA pueden trabajar de forma óptima a menores niveles de lux, sin percibir afectado su rendimiento, es decir, poseen mayor capacidad de adaptación a planos de trabajo más oscuros.

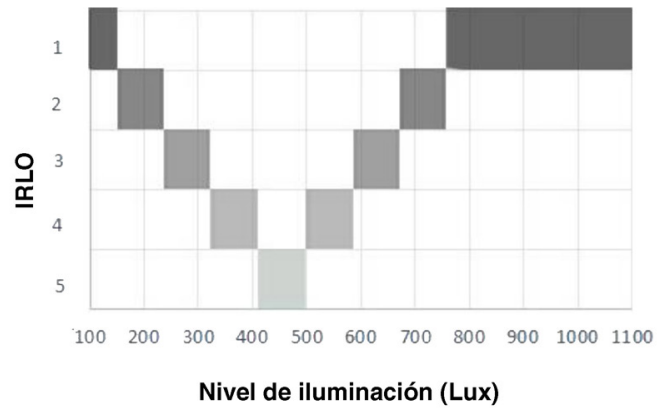

Figura 23. Rangos de variabilidad del RL influenciado por niveles de iluminación (lux) durante PVe en OTAA.

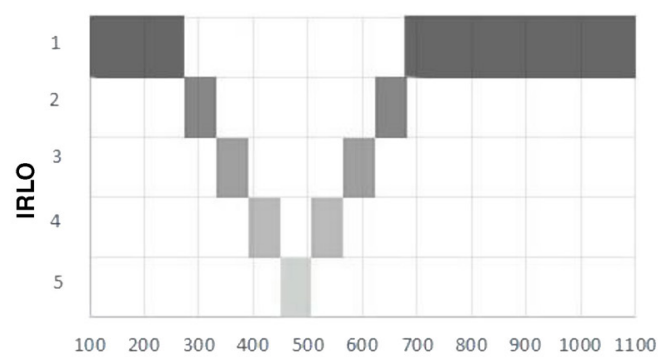

Nivel de iluminación (Lux)

Figura 25. Rangos de variabilidad del RL influenciado por niveles de iluminación (lux) durante PTr en OTAA.

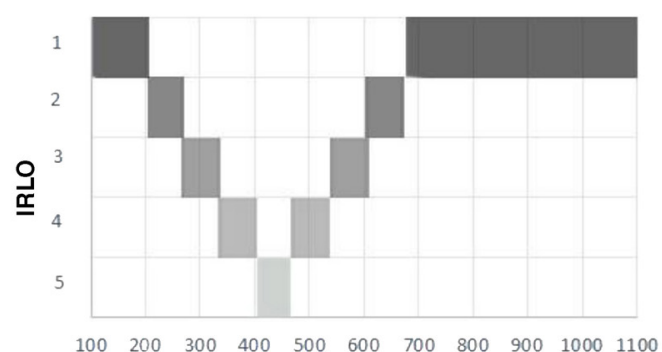

Nivel de iluminación (Lux)

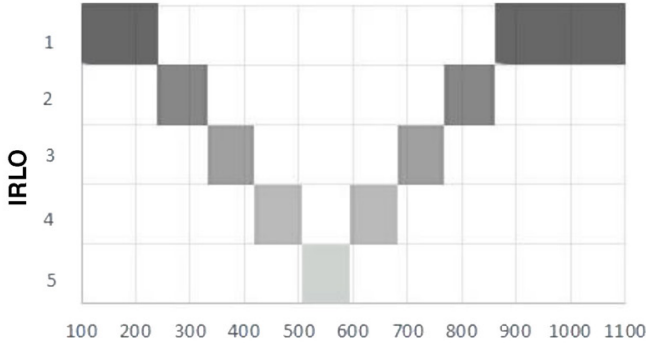

Nivel de iluminación (Lux)

Figura 24. Rangos de variabilidad del RL influenciado por niveles de iluminación (lux) durante PVe en OTAC.

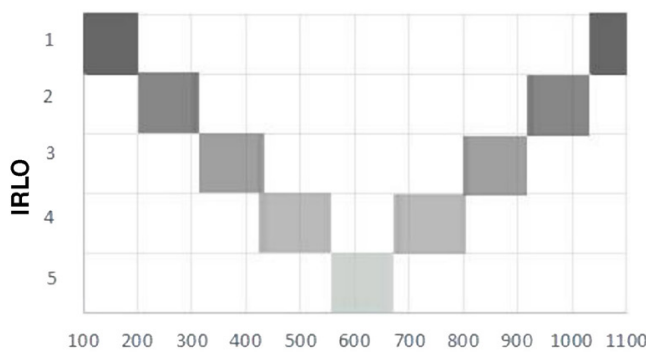

Nivel de iluminación (Lux)

Figura 26. Rangos de variabilidad del RL influenciado por niveles de iluminación (lux) durante PTr en OTAC.

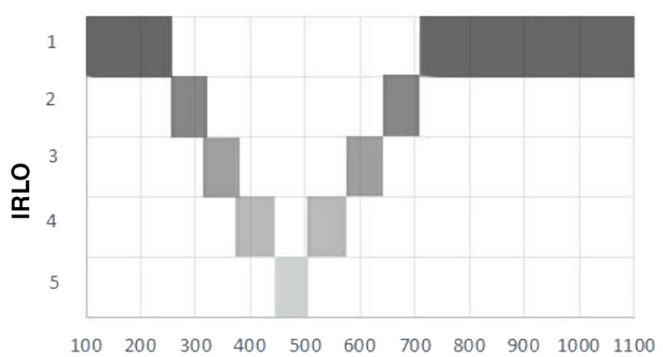

Nivel de iluminación (Lux)

Figura 28. Rangos de variabilidad del RL influenciado por niveles de iluminación (lux) durante PIn en OTAC. 


\subsection{Variable nivel de ruido}

El confort sonoro es afectado por el nivel de ruido cuando se trata de un sonido que provoque molestias, y se mide en potencia sonora con ponderación de tiempo lento y ponderación de frecuencia A (dBA, decibelio ponderado).

Las siguientes figuras muestran la clasificación de rangos de rendimiento obtenidos por periodo de estudio.

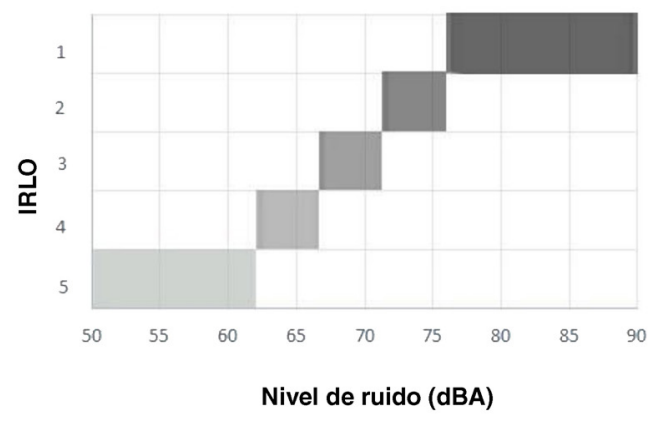

Figura 29. Rangos de variabilidad del RL influenciado por niveles sonoros (dBA) durante PVe en OTAA.

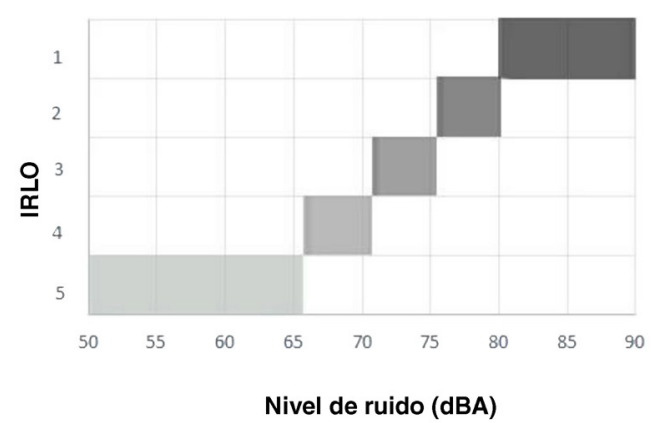

Figura 31. Rangos de variabilidad del RL influenciado por niveles sonoros (dBA) durante PTr en OTAA.

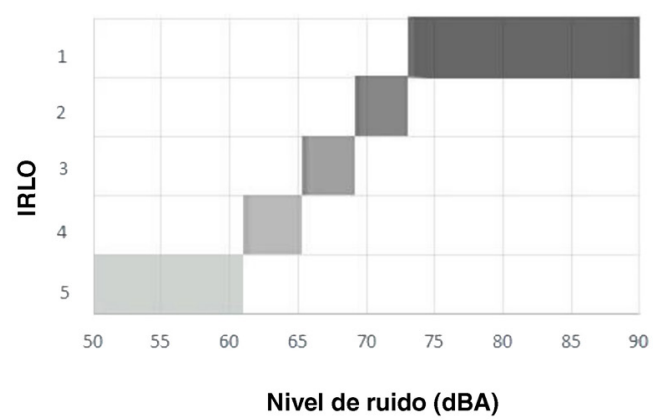

Figura 33. Rangos de variabilidad del RL influenciado por niveles sonoros (dBA) durante PIn en OTAA.
De los valores hallados correspondientes al parámetro Nivel de ruido, se detecta que los rangos propios en OTAA durante los tres periodos de estudio, presentan mayor amplitud respecto a OTAC, existiendo una diferencia de casi $5 \mathrm{dBA}$ entre ambas tipologías de oficina (ver figura 29 a 34).

Se reconoce al UT de OTAA con mayor capacidad de aceptar niveles de ruido elevados sin ver afectado su rendimiento laboral.

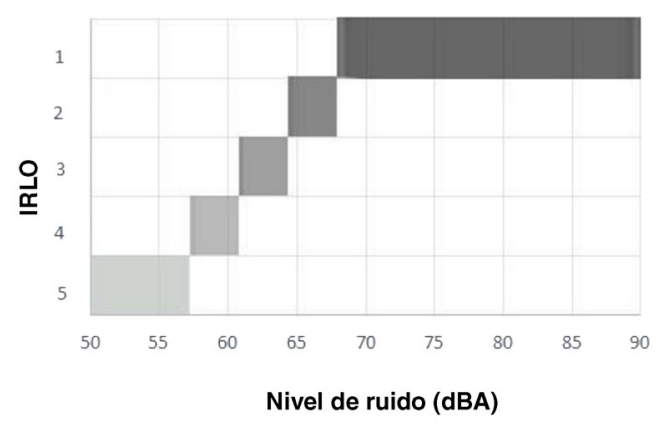

Figura 30. Rangos de variabilidad del RL influenciado por niveles sonoros (dBA) durante PVe en OTAC.

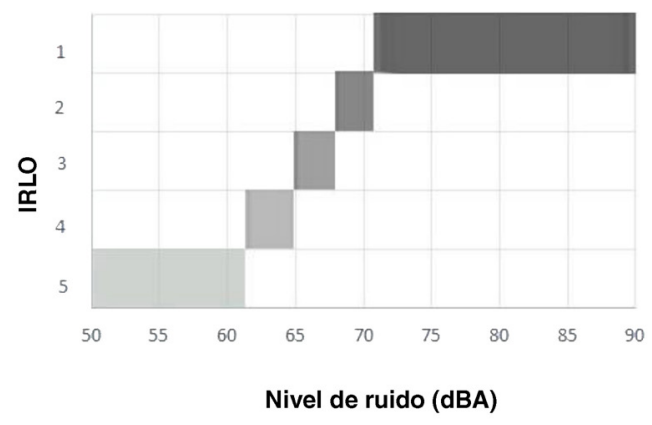

Figura 32. Rangos de variabilidad del RL influenciado por niveles sonoros (dBA) durante PTr en OTAC.

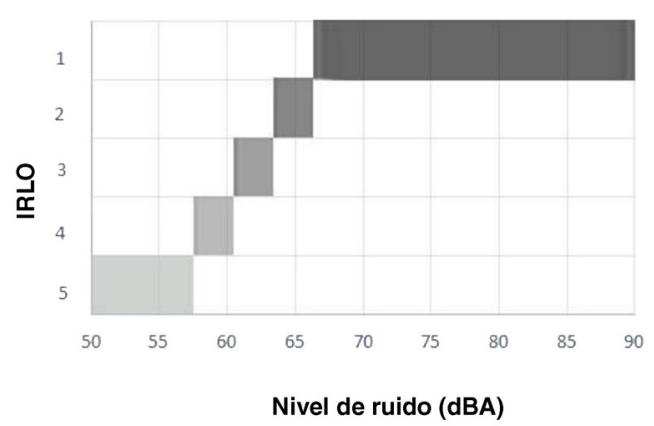

Figura 34. Rangos de variabilidad del RL influenciado por niveles sonoros (dBA) durante PIn en OTAC. 


\section{CONCLUSIONES}

De la construcción de los rangos de variabilidad es posible conocer desde las zonas más óptimas hasta las más vulnerables respecto al rendimiento del UT en zona templada cálida.

La construcción de los IRLO por cada variable de estudio y periodo del año, permite valorar al rendimiento laboral del UT, variando desde 1 (rendimiento malo/bajo) al 5 (rendimiento excelente/óptimo). De este modo un IRLO igual o cercano a 5, además de indicar las mejores condiciones ambientales para el óptimo rendimiento del UT en pos de la salud, supone un aporte "beneficioso" a las condiciones de comodidad/confort (térmico, visual, acústico y a la calidad del aire), siempre en vista de un uso racional de los recursos para el funcionamiento del edificio. Un IRLO igual o cercano a 1 (malo) indica al Buildingmanager tomar decisiones a tiempo, durante el curso del funcionamiento del edificio a fin de evitar patologías identificadas con la salud y la comodidad del UT, y no continuar en detrimento de las condiciones de calidad del UT y sus propias rentabilidades.

Del análisis tipológico-arquitectónico realizado, donde se observa que el UT sufre un proceso de adaptación continuo a rangos fluctuantes de variables ambientales de influencia, se afirma que la capacidad adaptativa ambiental del UT se encuentra vinculada al tipo de oficina que habita. Teniendo en cuenta las ventajas que presenta la OTAC frente a la OTAA respecto al mayor factor de ocupación, existencia de ventanas y su posibilidad de apertura, el UT desarrolla un mayor nivel de adaptación ambiental en espacios en desventaja como lo es la OTAA.

Las figuras 35 a 38 muestran las zonas deseables de alcanzar (rangos del $3 \mathrm{al} 5$ ) por variable de estudio, teniendo en cuenta el diseño espacial de oficina. El color verde oscuro corresponde a zona "excelente", verde medio para zona "muy buena" y verde claro para zona "buena" de rendimiento para el UT.

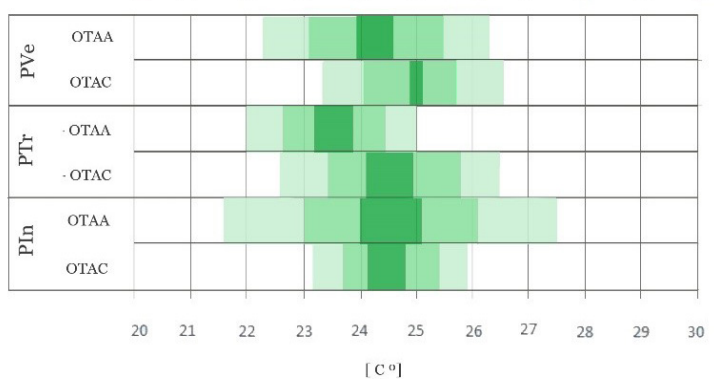

Figura 35. Zonas deseables a alcanzar (rango 3 al 5) correspondiente a confort térmico.

Esta herramienta es representativa de las condiciones de RL que se ofrecen en OTAA y OTAC en regiones con clima templados cálidos. A fin de extender su uso deberá emplearse la herramienta metodológica en otros casos de estudio a nivel local.

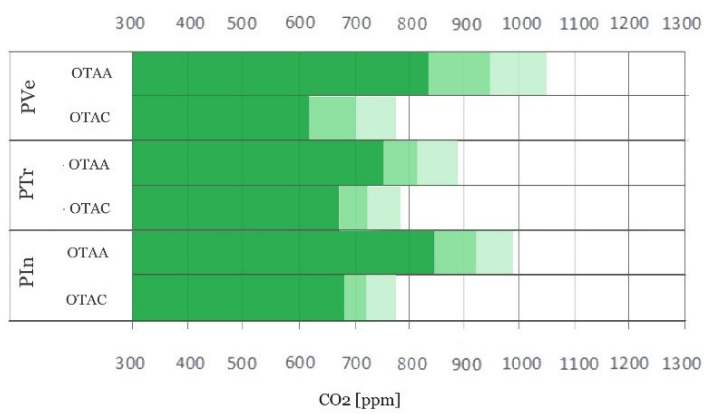

Figura 36. Zonas deseables a alcanzar (rango 3 al 5) correspondiente a calidad del aire.

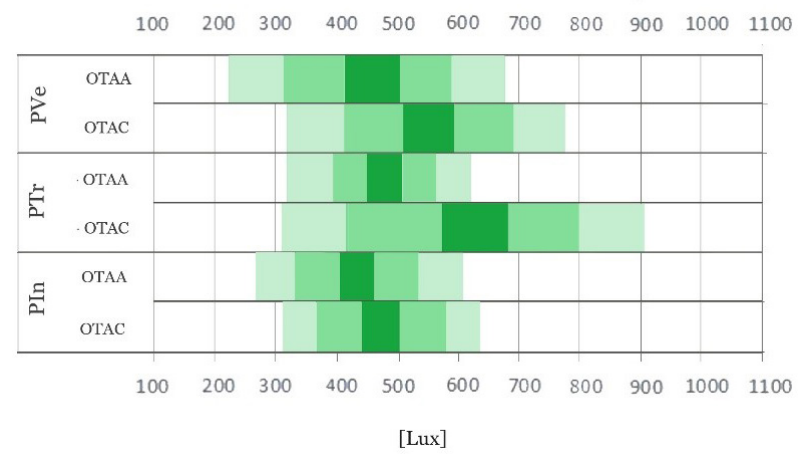

Figura 37. Zonas deseables a alcanzar (rango 3 al 5) correspondiente a confort lumínico.

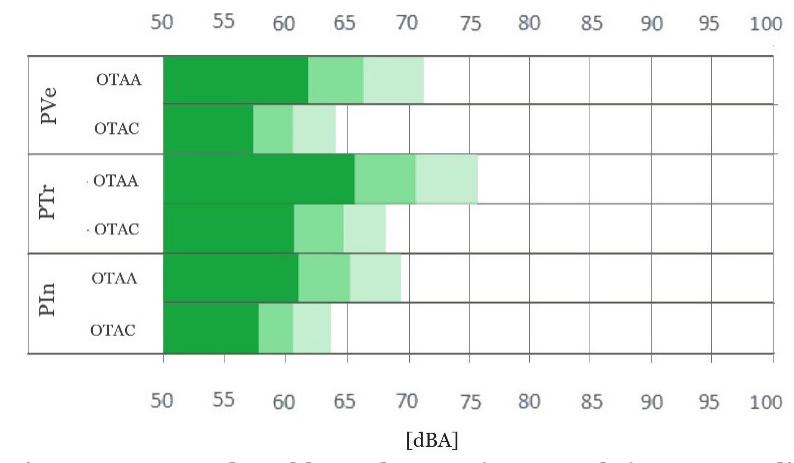

Figura 38. Zonas deseables a alcanzar (rango 3 al 5) correspondiente a confort acústico.

\section{AGRADECIMIENTOS}

Se agradece al CONICET (Concejo Nacional de Investigaciones Científicas y Tecnológicas de Argentina) y a la FAUDUNSJ (Facultad de Arquitectura, Urbanismo y Diseño - Universidad Nacional de San Juan), por el fomento a investigadores vinculados a este trabajo.

\section{REFERENCIAS}

(1) Tamers, S. L., Chosewood, L. C., Childress, A., Hudson, H., Nigam, J., \& Chang, C. C. (2019). Total Worker Health $®$ 2014-2018: the novel approach to worker safety, health, and well-being evolves. International journal of environmental research and public health, 16(3): 321. DOI: https://doi.org/10.339o/ijerph16030321

(2) Haynes, B., Suckley, L., \& Nunnington, N. (2017). Workplace productivity and office type: An evaluation of office occupier differences based on age and gender. Journal of Corporate Real Estate. DOI: https://doi.org/10.1108/JCRE11-2016-0037 
(3) Alker, J., Malanca, M., Pottage, C., \& O’Brien, R. (2014). Health, wellbeing \& productivity in offices: The next chapter for green building. World Green Building Council. Ver en linea en: https://www.worldgbc.org/sites/default/files/compressed_WorldGBC_Health_Wellbeing_Productivity_Full_Report_Dbl_Med_Res_Feb_2015.pdf

(4) Oficina Internacional del Trabajo (27 de Agosto 2018) Garantizar un tiempo de trabajo decente para el futuro. Estilo APA. Recuperado de https://www.ilo.org/wcmsp5/groups/public/---ed_norm/---relconf/documents/meetingdocument/wcms_618490.pdf.

(5) Wei, W., Wargocki, P., Zirngibl, J., Bendžalová, J., \& Mandin, C. (2020). Review of parameters used to assess the quality of the indoor environment in Green Building certification schemes for offices and hotels. Energy and Buildings, 209, 109683. DOI: https://doi.org/10.1016/j.enbuild.2019.109683

(6) Wargocki, P., \& Wyon, D. P. (2017). Ten questions concerning thermal and indoor air quality effects on the performance of office work and schoolwork. Building and Environment, 112, 359-366. DOI: https://doi.org/10.1016/j.buildenv.2016.11.020

(7) Lamb, S., \& Kwok, K. C. (2016). A longitudinal investigation of work environment stressors on the performance and wellbeing of office workers. Applied Ergonomics, 52, 104-111. DOI: https://doi.org/10.1016/j.apergo.2015.07.010

(8) Maula, H., Hongisto, V., Koskela, H., \& Haapakangas, A. (2016). The effect of cooling jet on work performance and comfort in warm office environment. Building and Environment, 104, 13-20. DOI: https://doi.org/10.1016/j.buildenv.2016.04.018

(9) Candanedo, L. M., \& Feldheim, V. (2016). Accurate occupancy detection of an office room from light, temperature, humidity and $\mathrm{CO}_{2}$ measurements using statistical learning models. Energy and Buildings, 112, 28-39. DOI: https://doi. org/10.1016/j.enbuild.2015.11.071

(10) Shriram, S., Ramamurthy, K., \& Ramakrishnan, S. (2019). Effect of occupant-induced indoor $\mathrm{CO}_{2}$ concentration and bioeffluents on human physiology using a spirometric test. Building and Environment, 149, 58-67. DOI: https://doi.org/10.1016/j.buildenv.2018.12.015

(11) Liu, T., Lin, C. C., Huang, K. C., \& Chen, Y. C. (2017). Effects of noise type, noise intensity, and illumination intensity on reading performance. Applied Acoustics, 120, 70-74. DOI: https://doi.org/10.1016/j.apacoust.2017.01.019

(12) Yang, W., \& Moon, H. J. (2019). Combined effects of acoustic, thermal, and illumination conditions on the comfort of discrete senses and overall indoor environment. Building and Environment, 148, 623-633. DOI: https://doi.org/10.1016/j.buildenv.2018.11.040

(13) Wu, H., Wu, Y., Sun, X., \& Liu, J. (2020). Combined effects of acoustic, thermal, and illumination on human perception and performance: A review. Building and Environment, 169, 106593. DOI: https://doi.org/10.1016/j.buildenv.2019.106593

(14) Liebl, A., \& Jahncke, H. (2017). Review of research on the effects of noise on cognitive performance 2014-2017. In 12th ICBEN conference on noise as a public health problem, 18-22 June 2017, Zurich, Switzerland. Ver en linea en: http:// www.icben.org/2017/ICBEN\%202017\%20Papers/SubjectArea04_Liebl_0401_4017.pdf

(15) Kari, T., Makkonen, M., \& Frank, L. (2017). The effect of using noise cancellation earplugs in open-plan offices on the offices on the work well-being and work performance of software professionals. In Mediterranean Conference on Information Systems (MCIS). Association For Information Systems. Ver en linea en: http://urn.fi/URN:NBN:fi:jyu-201712214857

(16) Norma IRAM 11603 (1996). Acondicionamiento térmico de edificios. Clasificación bioambiental de la República Argentina.

(17) Minetti, J. L., Carletto, P. M., \& SIERRA, A. P. Y. E. (1986). El régimen de precipitaciones de San Juan y su entorno. Centro de Investigaciones Regionales de San Juan.

(18) Puliafito, S. E., Allende, D. G., Mulena, C. G., Cremades, P., \& Lakkis, S. G. (2015). Evaluation of the WRF model configuration for Zonda wind events in a complex terrain. Atmospheric Research, 166, 24-32. DOI: https://doi.org/10.1016/j. atmosres.2015.06.011

(19) Perucca, L. P., \& Martos, L. M. (2012). Geomorphology, tectonism and Quaternary landscape evolution of the Central Andes of San Juan (30 S-69 W), Argentina. Quaternary International, 253, 80-90. DOI: https://doi.org/10.1016/j. quaint.2011.08.009

(20) ISO 7730 (1994). Moderate thermal environments-determinations of the PMV and PPD indces and specification of the conditions for thermal comfort. International Organization for Standardization (ISO).

(21) De Dear, R. (2004). Thermal comfort in practice. Indoor air, 14(s 7): 32-39. DOI: https://doi.org/10.1111/j.16000668.2004.00270.x

(22) Kuchen, E., \& Fisch, M. N. (2009). Spot monitoring: thermal comfort evaluation in 25 office buildings in winter. Building and Environment, 44(4): 839-847. DOI: https://doi.org/10.1016/j.buildenv.2008.06.023

(23) Humphreys, M. A., \& Nicol, J. F. (2007). Self-assessed productivity and the office environment: monthly surveys in five European countries. ASHRAE transactions, 113, 606. Ver en linea en: https://www.proquest.com/openview/8cfc5a0731a88d2407b3521e54829ebe/1?pq-origsite $=$ gscholar\&cbl $=34619$ 